J. Math. Biol. (1994) 33: 193-210

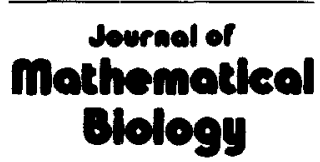

(C) Springer-Verlag 1994

\title{
Travelling waves in a tissue interaction model for skin pattern formation
}

\author{
G. C. Cruywagen ${ }^{1}$, P. K. Maini ${ }^{2}$, J. D. Murray ${ }^{1}$ \\ ${ }^{1}$ Department of Applied Mathematics FS-20, University of Washington, \\ Seattle, WA 98195 , USA \\ ${ }^{2}$ Centre for Mathematical Biology, Mathematical Institute, 24-29 St Giles', \\ Oxford, OX1 3LB, UK
}

Received 5 November 1993; received in revised form 28 February 1994

\begin{abstract}
Tissue interaction plays a major role in many morphogenetic processes, particularly those associated with skin organ primordia. We examine travelling wave solutions in a tissue interaction model for skin pattern formation which is firmly based on the known biology. From a phase space analysis we conjecture the existence of travelling waves with specific wave speeds. Subsequently, analytical approximations to the wave profiles are derived using perturbation methods. We then show numerically that such travelling wave solutions do exist and that they are in good agreement with our analytical results. Finally, the biological implications of our analysis are discussed.
\end{abstract}

Key words: Travelling waves - Tissue interaction - Perturbation procedure Wavespeed

\section{Introduction}

Travelling wavefronts are the precursors to a vast range of developmental processes seen in embryonic tissue. For example, both chemical and mechanical waves propagate on the surface of many vertebrate eggs shortly after fertilization. Furthermore, during morphogenesis, regular patterns often develop behind a frontier of pattern which travels across tissue.

Cheer et al. (1987) proposed a reaction-diffusion system to model the progression of calcium waves on the egg of the teleost fish Medaka. This model was extended by Lane et al. (1987) to account for cortical contraction waves which accompany the calcium waves. They coupled the mechanochemical system for epithelial cell movements, as proposed by Murray and Oster (1984), to a calcium conservation equation. 
Recently, Cruywagen and Murray (1992) proposed a novel model to account for the tissue interaction that leads to feather germ patterning in chick skin. In this paper we show that their model can similarly produce a contraction wave propagating through the epithelial layer. At this stage we only consider the one-dimensional version of the model. Waves in two-dimensions are quite different.

In Sect. 2 we present a brief review of the tissue interaction model and reduce it to an ordinary differential equation system by transforming into travelling wave coordinates. In Sect. 3 we carry out a linear analysis of this ordinary differential equation system and find conditions on the model parameters under which travelling wave solutions may be possible. A regular perturbation approximation to the travelling wave solutions is obtained in Sect. 4 while, in Sect. 5 , these perturbation solutions are compared with the numerical solutions of the system of ordinary differential equations and also to the system of partial differential equations. From the perturbation analysis and numerical results we make some biological interpretations and predictions; these are discussed in Sect. 6.

\section{The model}

Here we briefly describe the tissue interaction model and refer the reader to Cruywagen and Murray (1992) for full details. (For more details about modelling tissue interaction in general see Murray et al., 1993). The model assumes that tissue interaction between the epithelial and dermal skin layers is mediated by two signal chemicals which are secreted, respectively, in each layer and diffuse across the basal lamina which separates the layers. It consists of seven equations: four to describe the production, degradation, and diffusion of the chemicals within and between layers; two conservation equations for the dermal and epidermal cell densities, and finally, a force balance equation for modelling stresses in the epithelium. As the chemical equations are standard, we shall not describe them here but will, instead, focus on the force balance and cell density equations.

The epithelium is modelled as a visco-elastic continuum at low Reynolds number in which active traction forces exerted by the epidermal cells are balanced by elastic restoring forces and external elastic tethering to the basal lamina. The force balance equations then has the form

$$
\nabla \cdot\left\{\frac{E}{1+v}\left[\boldsymbol{\varepsilon}-\beta_{1} \nabla^{2} \boldsymbol{g}+\frac{v}{1-2 v}\left(\theta-\beta_{2} \nabla^{2} \theta\right) \boldsymbol{I}\right]+\mu_{1} \frac{\partial \boldsymbol{\varepsilon}}{\partial t}+\mu_{2} \frac{\partial \theta}{\partial t} \boldsymbol{I}+\tau \boldsymbol{S} \boldsymbol{I}\right\}=\rho \boldsymbol{u},
$$

where $\boldsymbol{u}(\boldsymbol{x}, t)$ is the displacement at time $t$ of a material point in the epithelium which was initially at position $x, \theta=\nabla \cdot \boldsymbol{u}$ is the dilation, $\varepsilon=\frac{1}{2}\left(\nabla \boldsymbol{u}+\nabla \boldsymbol{u}^{T}\right)$ is the strain tensor, where $T$ denotes the transpose, and $s(x, t)$ is the concentration of the signal chemical produced in the dermis. The parameters $E$ and $v$ are Young's modulus and Poisson's ratio respectively, while $\mu_{1}$ and $\mu_{2}$ are the 


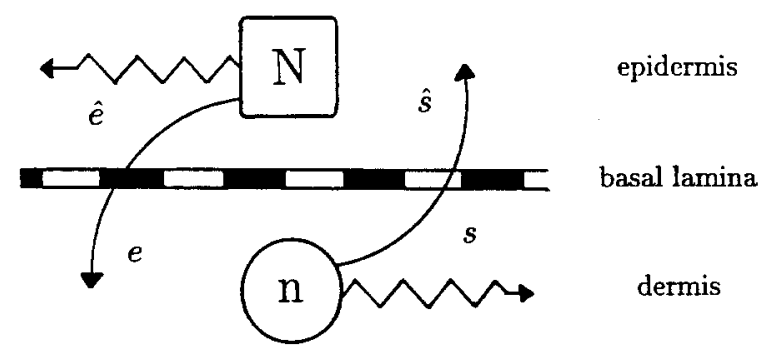

Fig. 1. A schematic diagram of the tissue interaction model of Cruywagen and Murray 1992. Dermal cells, $n$, secrete a signal chemical $s$, which diffuses into the epithelial layer. This chemical increases the traction produced by the epithelial cells $N$. The chemical $e$ is secreted by epithelial cells and diffuses into the dermis, where it acts as a chemoattractant for dermal cells

shear and bulk viscosities respectively, $I$ is the unit tensor, and $\beta_{1}$ and $\beta_{2}$ reflect long-range elastic stresses (see Murray 1989). For algebraic simplicity only, we have assumed here that the traction $\tau$ is a linear function of the dermal signal chemical, rather than the more complicated non-linear form used in Cruywagen and Murray (1992).

We assume that the epidermal cells move only due to convection, hence the epidermal cell density, $N(\boldsymbol{x}, t)$, satisfies the conservation equation

$$
\frac{\partial N}{\partial t}=-\nabla \cdot N \frac{\partial \boldsymbol{u}}{\partial t}
$$

The conservation equation for dermal cell density, $n(\boldsymbol{x}, t)$, accounts for diffusion, mitosis, and chemotaxis towards the signal chemical produced in the epithelium. Hence, the dermal chemotaxis equation is

$$
\frac{\partial n}{\partial t}=D \nabla^{2} n-\alpha \nabla \cdot n \nabla e+r n(\bar{n}-n),
$$

where $D$ is the coefficient of diffusion, $\alpha$ the chemotaxis coefficient, $e(x, t)$ the concentration of the signal chemical produced in the epithelium, and $r$ and $\bar{n}$ are positive constants (see Fig. 1 for a schematic diagram of the interaction mechanism).

We can linearise about the uniform steady state of $N$, say $\hat{N}$, and integrate (2.2) to obtain a linear relationship between $N$ and $\theta$, namely $N=\hat{N}(1-\theta)$. Using this and assuming that the cell kinetics occur on a fast timescale one can find actual algebraic expressions for the signal chemicals. From these expressions, in particular $s=n /(1+\nu(1-\theta))$ and $e=(1-\theta) /\left(1+\gamma^{n}\right)$, we observe that the active traction stress in the epidermis is induced by high concentrations of dermal cells, but inhibited by high concentrations of epithelial cells. Similarly, dermal chemotaxis occurs towards high concentrations of epithelial cells, but is inhibited by high concentrations of dermal cells. 
When nondimensionalised, the system takes the form, in one-dimension,

$$
\begin{gathered}
\mu \frac{\partial^{3} \theta}{\partial t \partial x^{2}}+\frac{\partial^{2} \theta}{\partial x^{2}}-\beta \frac{\partial^{4} \theta}{\partial x^{4}}+\frac{\partial^{2}}{\partial x^{2}}\left\{\frac{\tau n}{1+v(1-\theta)}\right\}=\rho \theta, \\
\frac{\partial n}{\partial t}=\frac{\partial^{2} n}{\partial x^{2}}-\alpha \frac{\partial}{\partial x}\left\{n \frac{\partial}{\partial x}\left(\frac{1-\theta}{1+\gamma n}\right)\right\}+n(1-n),
\end{gathered}
$$

where $\mu, \beta, \tau, \nu, \rho, \alpha$ and $\gamma$ are positive parameters.

The tissue interaction in these caricature equations is represented in $(2.4 \mathrm{a})$ by the fourth term on the left-hand side, in which cell traction in the epidermis is a function of dermal cell density, and in (2.4b) by the second term on the right-hand side in which dermal cell chemotaxis is a function of the dilation in the epidermis.

To analyse the system for travelling waves we look for solutions in the usual way by setting $z=x+c t$, where the constant $c$ is the positive wavespeed. We are thus concerned with waves moving in the negative $z$-direction. With this change of variables (2.4) converts to the following system of ordinary differential equations in $z$, where $n$ and $\theta$ are now functions of $z$,

$$
\begin{gathered}
\mu c \frac{d^{3} \theta}{d z^{3}}+\frac{d^{2} \theta}{d z^{2}}-\beta \frac{d^{4} \theta}{d z^{4}}+\tau \frac{d^{2}}{d z^{2}}\left\{\frac{n}{1+v(1-\theta)}\right\}=\rho \theta, \\
c \frac{d n}{d z}=\frac{d^{2} n}{d z^{2}}-\alpha \frac{d}{d z}\left\{n \frac{d}{d z}\left(\frac{1-\theta}{1+\gamma n}\right)\right\}+n(1-n) .
\end{gathered}
$$

Note that if we set $\alpha=0$, which is equivalent to ignoring epidermal to dermal interaction, (2.5b) decouples from (2.5a) and is, in fact, the well studied Fisher equation which exhibits travelling wave solutions with wavespeed $c \geqq 2$ (see, for example, Fife 1979, Murray 1989).

\section{Linear analysis}

To prove the existence of travelling wave solutions for the two-way tissue interaction system (2.5) is a non-trivial task, since one has to deal with a six-dimensional phase space. However, using a linear analysis about the steady states, we can conjecture under which conditions a trajectory corresponding to a travelling wave solution will be possible.

The tissue interaction system (2.5) has the steady states $\theta=0, n=0$ and $\theta=0, n=1$. Realistic waves require a heteroclinic orbit in $n$, connecting the steady state $n=0$ to $n=1$, and a homoclinic orbit in $\theta$, connecting the steady state $\theta=0$ to itself. By setting

$$
\theta_{0}=\theta, \quad \theta_{1}=\theta^{\prime}, \quad \theta_{2}=\theta^{\prime \prime}, \quad \theta_{3}=\theta^{\prime \prime \prime}, \quad n_{0}=n, \quad n_{1}=n^{\prime},
$$


we can rewrite the coupled pair of ordinary differential equations (2.5) as a sixth order system, namely

$$
\begin{aligned}
& \theta_{0}^{\prime}=\theta_{1}, \\
& \theta_{1}^{\prime}=\theta_{2}, \\
& \theta_{2}^{\prime}=\theta_{3}, \\
& \theta_{3}^{\prime}=\frac{1}{\beta}\left\{\mu c \theta_{3}+\theta_{2}-\rho \theta_{0}+\frac{\tau}{\sigma^{3}}\left[\sigma\left(\sigma n_{1}^{\prime}+v n_{0} \theta_{2}\right)+2 \tau v \theta_{1}\left(\sigma n_{1}+v n_{0} \theta_{1}\right)\right]\right\} \\
& n_{0}^{\prime}=n_{1}, \\
& n_{1}^{\prime}=\frac{1}{\zeta^{2}+\alpha \gamma n_{0}\left(1-\theta_{0}\right)}\left\{\zeta^{2}\left[c n_{1}+n_{0}\left(n_{0}-1\right)\right]-\alpha \gamma n_{1}\left(1-\theta_{0}\right)-\alpha \zeta\left(\theta_{1} n_{1}+n_{0} \theta_{2}\right)\right. \\
&\left.+\frac{2 \alpha \gamma n_{0} n_{1}}{\zeta}\left[\zeta \theta_{1}+\gamma n_{1}\left(1-\theta_{0}\right)\right]\right\}
\end{aligned}
$$

where

$$
\sigma=1+v\left(1-\theta_{0}\right), \quad \zeta=1+\gamma n_{0},
$$

and the expression (3.6f) for $n_{1}^{\prime}$ must be substituted into (3.6d).

We now investigate whether a trajectory connecting the steady state $S_{0}=(0,0,0,0,0,0)$ to the steady state $S_{1}=(0,0,0,0,1,0)$ is possible. As in the simpler Fisher case, we will show that the steady state $S_{0}$ has an unstable manifold and the steady state $S_{1}$ has a stable manifold.

First, we linearise about the trivial steady state $S_{0}$ and, in the usual way, look for solutions of the form $\boldsymbol{A} e^{\lambda z}$, where the vector $\boldsymbol{A}$ is a constant and the sign of $\mathscr{R} \lambda$ determines the stability of the steady state. Substituting this into the linearized system leads to a system of six simultaneous homogeneous equations. A non-trivial solution for this system exists only if $\lambda$ satisfies a sixth order polynomial, which includes as solution, the complex conjugate pair

$$
\lambda_{0 \pm}=\frac{c \pm \sqrt{c^{2}-4}}{2},
$$

with the eigenvectors of the form

$$
\left[\kappa, \kappa \lambda_{0 \pm}, \kappa \lambda_{0 \pm}^{2}, \kappa \lambda_{0 \pm}^{3}, 1, \lambda_{0 \pm}\right]
$$

where

$$
\kappa=\frac{\tau\left(c \lambda_{0 \pm}-1\right)}{(1+v)\left(\beta \lambda_{0 \pm}^{4}-\mu c \lambda_{0 \pm}^{3}-\lambda_{0 \pm}^{2}+\rho\right)} .
$$

The four other eigenvalues $\lambda_{i}, i=1,2,3,4$ satisfy

$$
\beta \lambda^{4}-\mu c \lambda^{3}-\lambda^{2}+\rho=0,
$$

with eigenvectors of the form

$$
\left[1, \lambda_{i}, \lambda_{i}^{2}, \lambda_{i}^{3}, 0,0\right] .
$$


By integrating (2.5b) with respect to $z$ and imposing appropriate boundary conditions (see Sect. 4), we can easily show that $c>0$. Hence the steady state $S_{0}$ will always have a linearly unstable manifold (from (3.7)) spanned by the eigenvectors corresponding to the positive eigenvalues in (3.7) and (3.9).

Biologically the dermal cell density $n_{0}$ is non-negative. Since the eigenvector (3.8), corresponding to the eigenvalue with the positive real part in (3.7), has an imaginary $n_{1}$-component if $c<2$, such unrealistic trajectories are possible. However, if $c \geqq 2$ then $n_{0} \geqq 0$ on all trajectories originating from $S_{0}$, since the eigenvectors (3.8) will have real $n_{1}$-components. Thus, similar to the Fisher equation, for realistic travelling wave solutions for the reduced tissue interaction model there is a lower bound on the wavespeed.

We can also derive a linearly valid condition on the model parameters for determining when the epithelial cell dilation solution will exhibit oscillatory behaviour in the vicinity of $S_{0}$. Using Descartes' rule of signs, since all the model parameters, as well as the wavespeed $c$ are positive, it follows that the polynomial (3.9) has either two or zero positive and either two or zero negative roots. To determine the three turning points (3.9) we set its first derivative equal to zero and solve for $\lambda$ to obtain

$$
\lambda_{ \pm}^{*}=\frac{3 \mu c+\sqrt{9 \mu^{2} c^{2}-32 \beta}}{8 \beta} \text { and } \lambda^{*}=0 .
$$

By substituting $\lambda_{+}^{*}$ into the polynomial (3.9), we find the condition under which it will have two real positive roots, that is

$$
512 \rho \beta^{3}-27 \mu^{4} c^{4}-144 \beta \mu^{2} c^{2}-16 \beta-\mu c\left(9 \mu^{2} c^{2}+32 \beta\right)^{\frac{3}{2}}<0 .
$$

Under this condition the eigenvalues in (3.9) with positive real part are real and therefore oscillatory behaviour in the model variable about $S_{0}$ will not occur. However, if condition (3.11) is not satisfied then the imaginary eigenvalues in (3.9) have positive real parts. In this case oscillatory trajectories in $\theta$ could shoot from the steady state $S_{0}$.

Linearising about the steady state $S_{1}$ we find eigenvalues

$$
\lambda_{ \pm}=\frac{(1+\gamma) c w \pm \sqrt{(1+\gamma)^{2} c^{2} w^{2}+4(1+\gamma) w}}{2}
$$

as well as the four roots satisfying

where

$$
\beta \lambda^{4}-\mu \mathrm{c} \lambda^{3}-\zeta \lambda^{2}+\rho=0,
$$

$$
w=\frac{1+\gamma}{(1+\gamma)^{2}+\alpha \gamma}>0
$$

and

$$
\zeta=1+\frac{\tau v}{(1+v)^{2}}
$$


It is easy to see from (3.12) that $S_{1}$ always has a stable manifold for positive values of $c$. Since the eigenvalues (3.12) have no imaginary parts there will be no oscillation in cell density $n$, in the vicinity of the steady state.

Furthermore, as the polynomial (3.13), according to Descartes' rule of signs, has two or zero negative roots we can again find a linearly valid condition under which the dilation solution $\theta$, would not approach the zero steady state solution in an oscillatory manner. Using a similar method as before we can find the condition as

$$
512 \rho \beta^{3}-27 \mu^{4} c^{4}-144 \beta \zeta \mu^{2} c^{2}-16 \beta \zeta^{2}+\mu c\left(9 \mu^{2} c^{2}+32 \zeta \beta\right)^{\frac{3}{2}} \leqq 0 .
$$

If this condition is violated oscillatory trajectories in $\theta$ in the vicinity of $S_{1}$ are possible.

From the above analysis we conjecture that a biologically realistic travelling wave, connecting the steady states $S_{0}$ and $S_{1}$ for all parameter ranges could exist if $c \geqq 2$. In the following sections we shall examine by analytical and numerical means whether such travelling wave solutions actually do exist.

\section{Regular perturbation solution}

In this section we find a regular perturbation solution for the travelling wave solutions of the tissue interaction system (2.5). For the purpose of our perturbation method we assume that there does indeed exist a travelling wave solution for (2.5) satisfying the boundary conditions

$\lim _{z \rightarrow \infty} n(z)=1, \lim _{z \rightarrow-\infty} n(z)=0, \lim _{z \rightarrow \pm \infty} \theta(z)=\theta^{\prime}(z)=\theta^{\prime \prime}(z)=\theta^{\prime \prime \prime}(z)=n^{\prime}(z)=0$

We begin by rescaling the system of ordinary differential equations (2.5). To find the appropriate scalings we rescale the dependent and independent variables in $(2.5)$ by setting

$$
\Theta=\Sigma^{-1} \theta, \quad N=\phi n, \quad \xi=\delta z,
$$

where $\Theta, N$ and $\xi$ are the rescaled variables, $\delta$ is an appropriate scale for the independent variable and $\Sigma$ and $\phi$ are appropriate scales for the dependent variables.

Since we have no a priori reason for favouring one term above another we try to employ a scaling which simplifies (4.15) the least, that is, which makes as many terms as possible in the relevant differential equation have the same order of magnitude.

Using (4.16) gives, for (2.5a), after multiplying by $\Sigma$,

$$
\mu c \delta^{3} \Sigma \frac{d^{3} \Theta}{d \xi^{3}}+\delta^{2} \Sigma \frac{d^{2} \Theta}{d \xi^{2}}-\beta \delta^{4} \Sigma \frac{d^{4} \Theta}{d \xi^{4}}+\tau \delta^{2} \phi \frac{d^{2}}{d \xi^{2}}\left\{\frac{N}{1+\nu(1-\Sigma \Theta)}\right\}=\rho \Sigma \Theta .
$$


We find here that it is impossible to make all the terms the same order of magnitude, since there are five terms but only three free parameters $(\Sigma, \phi$ and $\delta$ ), and we are forced to favour some terms above others.

To ensure that we have a non-zero solution for this equation at the lowest order we must assume that the interaction term, in which $n(z)$ appears, is in the lowest order equations. Biological considerations suggest that the viscous and long range elastic terms, the first and third terms in (4.17), respectively are not as important as the short range elastic term and the epithelial tethering term, the second term and the term on the right hand side of (4.17), respectively.

We therefore assume that the interaction and the tethering terms are of the same order of magnitude, thus setting $\delta^{2} \tau \phi=\rho \Sigma$. This determines a unique relationship between $\delta$ and $\Sigma$ which would be violated if we assume other terms in the equation are also of the same order. Alternatively, one could assume that the elastic and the interaction terms are of the same order of magnitude. However, by using a similar procedure as we do below, exactly the same perturbation solutions for $n$ and $\theta$ result.

By using the scaling (4.16), where $\phi=1,(2.5 \mathrm{~b})$ becomes

$$
c \delta \frac{d N}{d \xi}=\delta^{2} \frac{d^{2} N}{d \xi^{2}}-\alpha \delta^{2} \frac{d}{d \xi}\left\{N \frac{d}{d \xi}\left(\frac{1-\Sigma \Theta}{1+\gamma \phi N}\right)\right\}+N(1-\phi N),
$$

which is the Fisher equation with an interaction term included. Here again we have to favour some terms above others. Murray (1977) gives a perturbation solution for the normal Fisher equation in terms of the small parameter $1 / \mathrm{c}^{2}$, by assuming that $\phi=1$ and that the term on the left hand side of the equation is the same order of magnitude as the logistic growth term on the right hand side. We make the same assumption for our interaction system, thus setting $c \delta=1$. From now on we shall again use $n$ instead of $N$.

So, if we assume that

$$
\delta=\frac{1}{c}, \quad \Sigma=\frac{\tau}{\rho c^{2}},
$$

and set $\varepsilon=\delta^{2}$ we obtain the rescaled system

$$
\begin{gathered}
\mu \varepsilon \frac{d^{3} \Theta}{d \xi^{3}}+\varepsilon \frac{d^{2} \Theta}{d \xi^{2}}-\varepsilon^{4} \beta \frac{d^{4} \Theta}{d \xi^{4}}+\rho \frac{d^{2}}{d \xi^{2}}\left\{\frac{n}{1+v(1-\varepsilon \tau \Theta / \rho)}\right\}=\rho \Theta . \\
\frac{d n}{d \xi}=\varepsilon \frac{d^{2} n}{d \xi^{2}}-\varepsilon \alpha \frac{d}{d \xi}\left\{n \frac{d}{d \xi}\left(\frac{1-\varepsilon \tau \Theta \rho}{1+\gamma n}\right)\right\}+n(1-n)
\end{gathered}
$$

with boundary conditions

$$
\begin{aligned}
& \lim _{\xi \rightarrow \infty} n(\xi)=1, \lim _{\xi \rightarrow-\infty} n(\xi)=0, \\
& \lim _{\xi \rightarrow \pm \infty} \Theta(\xi)=\Theta^{\prime}(\xi)=\Theta^{\prime \prime}(\xi)=\Theta^{\prime \prime \prime}(\xi)=n^{\prime}(\xi)=0 .
\end{aligned}
$$

These equations are translation-invariant in $\xi$, so for uniqueness we additionally fix one point,

$$
n(0)=\frac{1}{2} \text {. }
$$


The linear analysis of the previous section predicted that if the tissue interaction system has travelling wave solutions then their speed $c \geqq 2$, so that $\varepsilon \leqq 0.25$. Hence, we use a regular perturbation method to obtain approximate solutions to this system with $\varepsilon$ as our small parameter. Although $\varepsilon$ is relatively large when the wavespeed has minimum value $c=2$, a similar method gives very good perturbation solutions to the Fisher equation (see Murray 1977). We therefore assume that the solution of (4.18), satisfying the boundary conditions (4.19) and (4.20), can be written as a series expansion of the form

$$
\begin{aligned}
& \Theta(\xi, \varepsilon)=\Theta_{0}(\xi)+\varepsilon \Theta_{1}(\xi)+\ldots \\
& n(\xi, \varepsilon)=n_{0}(\xi)+\varepsilon n_{1}(\xi)+\ldots
\end{aligned}
$$

Substituting (4.21) into (4.18) reduces the system of nonlinear equations (4.18) to a hierarchy of linear equations. The boundary conditions at $\xi= \pm \infty$ and the choice of $n(0)=1 / 2$, which requires that $n(0, \varepsilon)=1 / 2$ for all $\varepsilon$, lead to the following conditions on $\Theta_{i}(\xi)$ and $n_{i}(\xi)$,

$$
\begin{array}{lll}
\lim _{\xi \rightarrow \pm \infty} \Theta_{i}(\xi)=0, & \lim _{\xi \rightarrow \pm \infty} \Theta_{i}^{\prime}(\xi)=0, \quad \text { for } i=0,1,2, \ldots \\
\lim _{\xi \rightarrow-\infty} n_{0}(\xi)=0, & \lim _{\xi \rightarrow \infty} n_{0}(\xi)=1, \quad n_{0}(0)=1 / 2, \\
\lim _{\xi \rightarrow \pm \infty} n_{i}(\xi)=0, & n_{i}(0)=0, \quad \text { for } i=1,2,3, \ldots
\end{array}
$$

Before carrying out the perturbation procedure we must decide on the magnitude of the physical model parameters $\mu, \beta, \tau, \nu, \rho, \alpha$ and $\gamma$. As these parameters are difficult to measure, we scale them so that the most significant biological terms have the same order of magnitude in the relevant equations. This way we keep as much flexibility as possible.

We assume that $\rho$ is $O(1)$. If we assume that $\alpha, \gamma$ or $v$ is $O(1 / \varepsilon)$ or larger, it becomes analytically intractable to find perturbation solutions for the system. Thus we assume that they are all $O(1)$. Biologically we expect the long range elasticity, reflected by $\beta$, and the viscous effects, measured by the parameter $\mu$, to be small and so, to insure that these effects do not dominate the short range elastic forces, we choose both $\beta$ and $\mu$ to be $O(1)$ or smaller. We also do not expect the chemically induced traction force, measured by the parameter $\tau$, to be larger than the restoring forces of the adhesion tethers, reflected by $\rho$, thus $\rho / \tau$ is $O(1)$ or larger. Later we shall discuss how the order of magnitude of these parameters may influence the solutions.

At $O(1)$ the equations are

$$
\begin{gathered}
\Theta_{0}=\frac{1}{1+v} \frac{d^{2} n_{0}}{d \xi^{2}} \\
\frac{d n_{0}}{d \xi}=n_{0}\left(1-n_{0}\right) .
\end{gathered}
$$


Integrating the second equation and using the midpoint condition $n(0)=1 / 2$ we find that

$$
n_{0}=\frac{e^{\xi}}{1+e^{\xi}}
$$

Substituting this into the first equation gives

$$
\Theta_{0}=\frac{e^{\xi}\left(1-e^{\xi}\right)}{(1+v)\left(1+e^{\xi}\right)^{3}} .
$$

Note that $\Theta_{0}$ satisfies the boundary conditions.

At $O(\varepsilon)$ we have

$$
\begin{gathered}
\Theta_{1}=\frac{\mu}{\rho} \frac{d^{3} \Theta_{0}}{d \xi^{3}}+\frac{1}{\rho} \frac{d^{2} \Theta_{0}}{d \xi^{2}}+\frac{1}{1+v} \frac{d^{2} n_{1}}{d \xi^{2}}+\frac{v}{(1+v)^{2}} \frac{d^{2}\left(n_{0} \Theta_{0}\right)}{d \xi^{2}} \\
\frac{d n_{1}}{d \xi}+\left(2 n_{0}-1\right) n_{1}=\frac{d^{2} n_{0}}{d \xi^{2}}-\alpha \frac{d}{d \xi}\left\{n_{0} \frac{d}{d \xi}\left(\frac{1}{1+\gamma n_{0}}\right)\right\}
\end{gathered}
$$

By substituting $n_{0}$ into (4.26b) and integrating, we have

$$
\begin{aligned}
n_{1}(\xi)= & f(\xi)\left[\xi-2 \ln \left(1+e^{\xi}\right)+k\right]-\frac{\alpha \gamma f(\xi)}{2(1+\gamma)^{2} h^{2}(\xi)} \\
& \times\left\{3+2 \gamma+2 h^{2}(\xi) \ln h(\xi)+h^{\prime}(\xi)\left[2-2 h(\xi)-h^{\prime}(\xi)\right]\right\},
\end{aligned}
$$

where

$$
f(\xi)=\frac{e^{\xi}}{\left(1+e^{\xi}\right)^{2}}, \quad h(\xi)=1+(1+\gamma) e^{\xi}
$$

the prime denotes the derivative with respect to $\xi$ and $k$ is the constant of integration. Using the midpoint condition, $n_{1}(0)=0$, we find that the integration constant is given by

$$
k=2 \ln 2+\frac{\alpha \gamma}{2(1+\gamma)^{2}(2+\gamma)^{2}}\left[2(2+\gamma)^{2} \ln (2+\gamma)-\gamma\left(3 \gamma^{2}+4\right)\right] .
$$

Substituting $n_{0}, n_{1}, \Theta_{0}$ and their relevant derivatives into (4.26a) we can find $\Theta_{1}$.

We can continue with this process to find expressions for the higher order terms, but the expressions become algebraically cumbersome. Besides, the additional correction provided by, say, the $O\left(\varepsilon^{2}\right)$ term, is too small to be significant.

Biologically it is very difficult to measure the dilation $\theta$, of the epithelial layer. However, since the relationship between the dilation and the displacement $u$ for the case of small strains is

$$
\frac{\partial u(x, t)}{\partial x}=\theta(x, t)
$$


we can find the perturbation solution in terms of the displacement $u$ by integrating the dilation function. In terms of the variable $\xi$, we have that

$$
\frac{d u(\xi)}{d \xi}=\frac{\tau}{\rho c} \Theta(\xi)
$$

The constant of integration can be set to zero if we assume that the displacement at infinity is zero. Assuming that we can integrate the perturbation solution for $\Theta(\xi)$ we find that

where

$$
u(\xi)=\frac{\tau}{\rho}\left(\varepsilon^{1 / 2} u_{0}(\xi)+\varepsilon^{3 / 2} u_{1}(\xi)+\ldots\right),
$$

$$
\begin{gathered}
u_{0}(\xi)=\frac{e^{\xi}}{(1+v)\left(1+e^{\xi}\right)^{2}}, \\
u_{1}(\xi)=\frac{\mu}{\rho} \Theta_{0}^{\prime \prime}+\Theta_{0}^{\prime}\left[\frac{1}{\rho}+\frac{v n_{0}(\xi)}{(1+v)^{2}}\right]+\frac{n_{1}^{\prime}(\xi)}{1+v}+\frac{v \Theta_{0} n_{0}^{\prime}(\xi)}{(1+v)^{2}} .
\end{gathered}
$$

From these expressions we see that $u(-\infty)=0$, at least to $O\left(\varepsilon^{3 / 2}\right)$. Extensive numerical simulation suggests that $u$ is zero as $z$ tends to $-\infty$.

Hence we have found a regular perturbation solution for the system of ordinary differential equations (2.5) when all the parameters are $O(1)$. The solution, however, changes if the parameter values do not satisfy this condition. We discuss this in the following two sections.

\section{Numerical solutions}

Here we solve the ordinary differential equation system (2.5) numerically and compare the results with the perturbation solutions obtained in the previous section. We solved (2.5) with NAG FORTRAN routine D02RAF which uses a finite difference technique for solving boundary value problems. An initial estimate is corrected using Newton iteration and deferred correction. We specified as initial condition the first order perturbation solutions (4.24) and (4.25) as obtained in the previous section.

The equations were solved on the spatial domain $[-20,20]$, which is large in comparison with the part of the domain where the solution rapidly changes shape. So as to satisfy the uniqueness criteria used in the previous section, the numerical solutions were shifted so that $n(0)=1 / 2$.

Our numerical simulations showed that biologically realistic waves occur only when the wavespeed satisfied $c \geqq 2.0$. In all the cases we computed with $c<2$, the dermal cell density dropped below zero in the region of the boundary $z=-20$. This confirms the predictions of the linear analysis of Sect. 3 .

To find the displacement $u(z)$, we numerically solved $u^{\prime}(z)=\theta(z)$ by using NAG FORTRAN routine D02CBF which integrates a first order, variable step, Adams routine. Since we require the displacement to be zero at $\pm \infty$ we 
assume as initial condition that $u(-20)=0.0$ and integrated to $z=20$. The numerical solution of $\theta(z)$ was calculated at discrete mesh points by the finite difference routine. Since the Adams routine requires the value for $\theta(z)$ at intermediate points we used NAG FORTRAN routine E01AAF to interpolate the value of $\theta(z)$ at the $z$-values required by the Adams routine, using the four closest mesh values of $\theta$. This routine uses Aitken's technique of successive linear interpolations.

We illustrate our numerical results with two examples for different values of the wavespeed $c$.

Example 1. We assumed that all our parameters are $O(1)$ so that we can compare our numerical solution with the regular perturbation solution. We therefore set $\mu, \beta, \tau, v, \rho, \alpha$ and $\gamma$ all equal to 1.0. It was further assumed that the wave travels at minimum speed, $c=2.0$, as predicted by the linear analysis of Sect. 3. For such a low value of the wavespeed $c$, the value of the small parameter $\varepsilon$ in our perturbation expansions (4.21) is relatively large.

The numerical solution is compared with the zero and first order analytical approximations in Fig. 2. We see that the approximation for $\theta$ improves
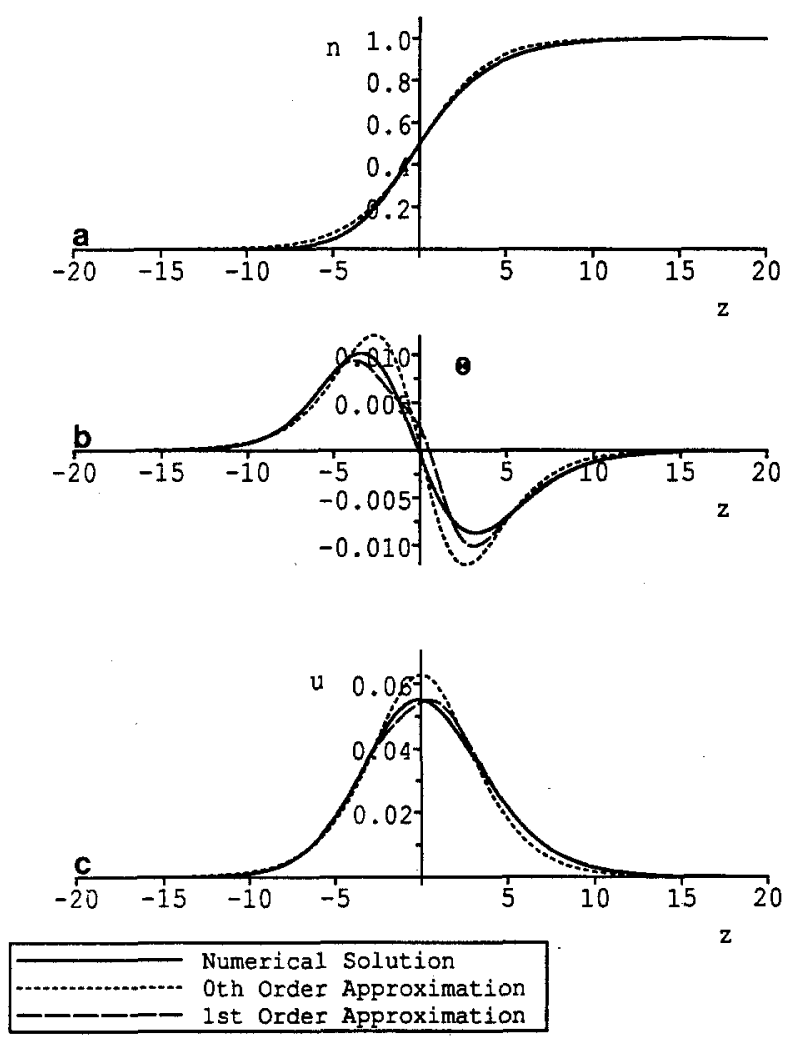

Fig. 2. A comparison of the numerical solution with the regular perturbation solutions to $O(1)$ and $O(\varepsilon)$ when $c=2$ and thus $\varepsilon=1 / 4$. The parameters are as in Example 1 
slightly by adding the $O(\varepsilon)$ term. The bend appearing in the solution at $z=0$ is due to the $\Theta^{\prime \prime}$ and $\Theta^{\prime \prime \prime}$ terms in our perturbation solution which are relatively large at the origin, see Fig. 3.

Example 2. Here we used the same parameter set as in Example 1, but we increased the wavespeed to $c=3$ so that $\varepsilon=1 / 9$. With the first order terms included the perturbation solution and numerical solution are almost identical, see Fig. 4.

In both examples considered above, the linear non-oscillatory conditions on $\theta$ in the vicinity of the steady states, (3.11) and (3.14), were satisfied. As expected the dilation solution approached the steady states smoothly. However, we also considered some examples with parameter sets so that conditions (3.11) and (3.14) were violated. In these cases the dilation solution $\theta$ changed sign as it approached the steady states, suggesting a possible oscillatory behaviour.

The full partial differential equation system (2.4) was solved numerically using NAG FORTRAN routine D03PGF, which discretises the spatial derivatives and solves the resulting system of ordinary differential equations using Gear's Method. We imposed zero flux boundary conditions. For the initial conditions we refer again to the Fisher case. The velocity of the travelling wavefront for the classical Fisher equation depends on the initial conditions. Kolgomoroff et al. (1937) proved that if the initial conditions satisfy

$$
n(x, 0)=\left\{\begin{array}{l}
1 \text { if } x \geqq x_{2} \\
0 \text { if } x \leqq x_{1}
\end{array},\right.
$$

where $x_{1}<x_{2}$ and $n(x, 0)$ is continuous in the domain $\left(x_{1}, x_{2}\right)$, then the solution of the Fisher equation evolves to a travelling wavefront solution with minimum speed $c=2.0$. We conjecture that this result also holds for our system.
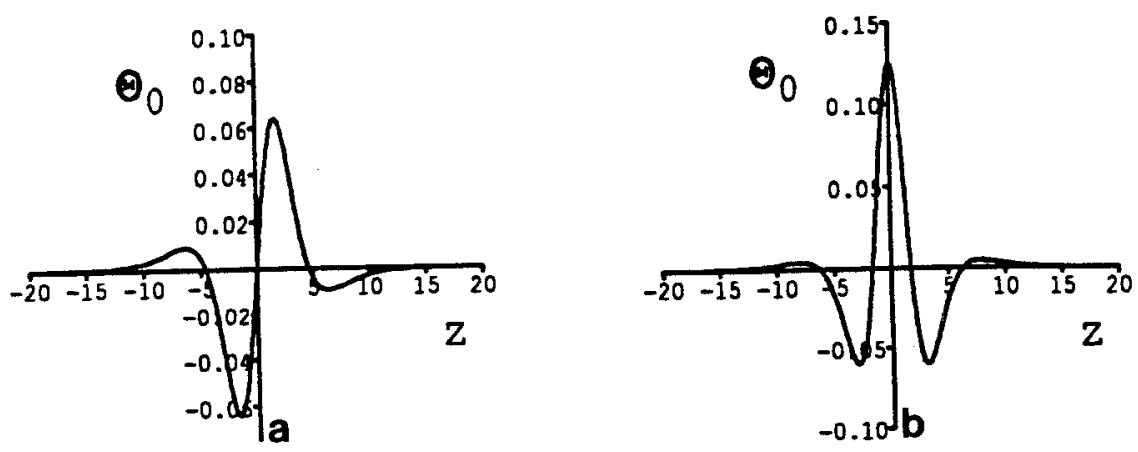

Fig. 3a, b. The contributions of the $\Theta_{0}^{\prime \prime}$ (a) and $\Theta_{0}^{\prime \prime \prime}$ (b) terms in the regular perturbation solution when parameters are as in Example 1 

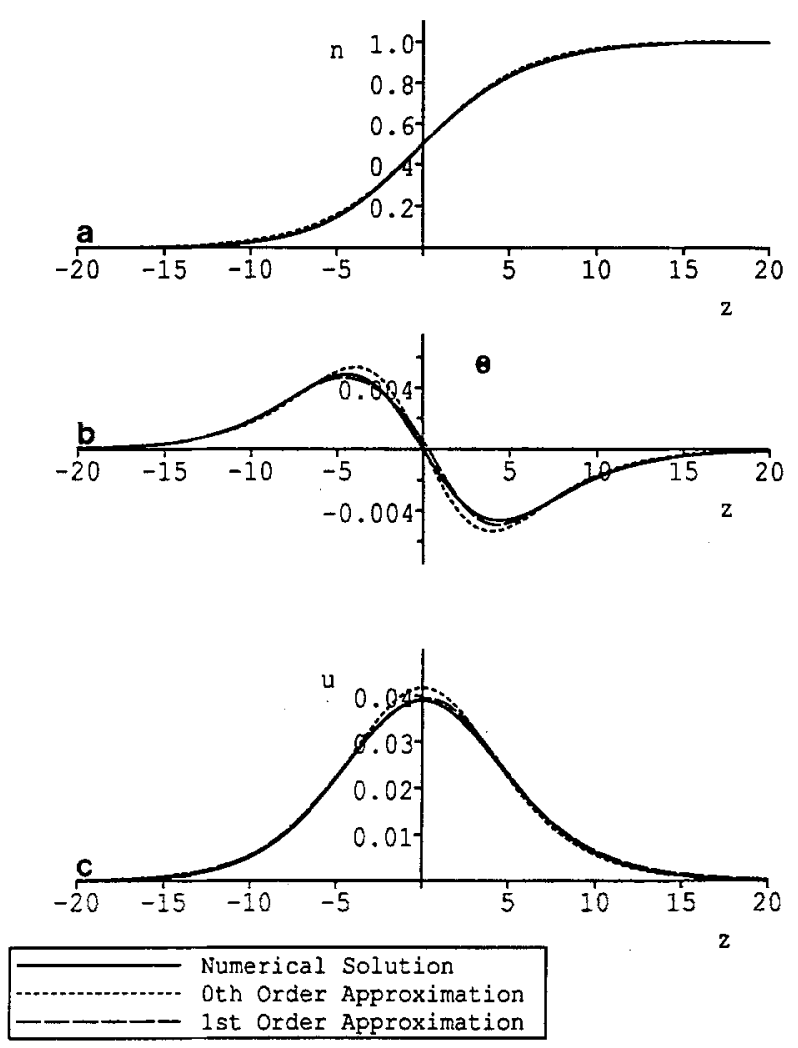

Fig. 4a-c. A comparison of the numerical solution with the regular perturbation solutions to $O(1)$ and $O(\varepsilon)$ when $c=3$ and thus $\varepsilon=1 / 9$. The parameters are as in Example 2

It is also known that for initial data other than the above, the wavespeed of the Fisher equation depends critically on the behaviour of $n(x, 0)$ as $x \rightarrow \pm \infty$. To find the dependence of the wavespeed of our system on the initial conditions at infinity, analysis similar to that proposed by Mollison (1977) for the Fisher equation is used.

We consider the leading edge of the evolving wave. Here, since $n$ and $\theta$ are small, we linearize the dermal cell equation to

$$
\frac{\partial n}{\partial t}=\frac{\partial^{2} n}{\partial x^{2}}+n
$$

which is similar to the linearized version of the Fisher equation at the leading edge. Assume that the initial condition in dermal cell density is

$$
n(x, 0)=A e^{a x} \quad \text { as } x \rightarrow-\infty,
$$

where $A$ and $a$ are positive arbitrary constants. We look for travelling wave solutions satisfying (5.30) and of the form

$$
n(x, t)=A e^{a(x+c t)},
$$


which represents the leading edge of the wavefront solution of the nonlinear equation. Substituting this expression into the linear equation (5.30) gives the dispersion relation

$$
c=a+\frac{1}{a} .
$$

So, for $a=1$, the wavespeed $c$ takes the minimum value 2 . For all other positive values of $a, c$ is larger than 2 .

Now consider $\min \left[e^{a x}, e^{x}\right]$ for $x$ large and negative. If $a<1$ then $e^{a x}>e^{x}$ for $x<0$ and therefore the velocity of propagation will depend on the leading edge of the wave and the value of $c$ is given by (5.31). On the other hand if $a>1$ then $e^{a x}$ is bounded from above by $e^{x}$ and thus the wavefront with speed $c=2$. So

$$
c= \begin{cases}a+1 / a & \text { if } 0<a \leqq 1 \\ 2 & \text { if } a \geqq 1\end{cases}
$$
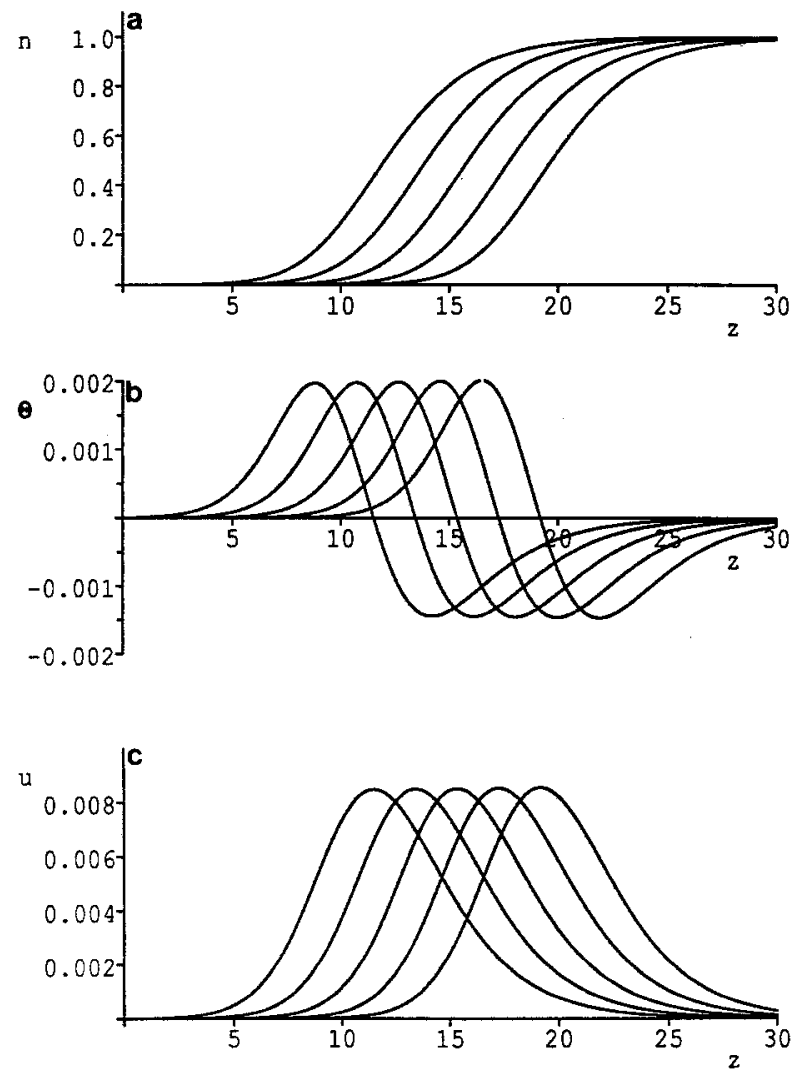

Fig. 5a-c. Time evolution of the travelling front at intervals of unit time when parameters are as in Example 3 and initial conditions as in (5.29). The wave velocity, $c=2.0$, is as predicted by the linear analysis 
We now use this result to construct initial conditions for the numerical simulations.

Solving the partial differential equation system with parameter values as in Example 1 and initial conditions (5.29), excellent agreement was obtained with the solution of the system of ordinary differential equations (2.5). As expected, we found that the wave solution did indeed travel at minimum speed $c=2.0$.

Example 3. Here we solve the partial differential equation system with the most realistic parameter set

$$
\mu=0.1, \quad \beta=0.01, \quad \tau=1.0, \quad v=\frac{1}{3}, \quad \rho=15.0, \quad \alpha=1.0, \quad \gamma=\frac{1}{3} .
$$

We have chosen $\beta$ to be very small since we expect the long range elastic forces in the mechanical balance equation to be small. As before we assumed that $n(x, 0)$ satisfies (5.29). As expected, a wavespeed $c=2.0$, was obtained (see Fig. 5). This confirms the predictions made from the analysis of Sect. 3.

Example 4. We use the same parameter set as in the previous example, but set our initial conditions so that a wavespeed $c=5.2$ is expected. According to (5.32) this means that the initial conditions at the leading edge must be of the form $n(x, 0)=e^{5 x}$. To simulate this numerically the dermal leading edge was perturbed accordingly after each numerical iteration. (We assumed the leading edge to be the region where the dermal cell density is less than 0.1). A wavespeed $c=5.2$ was indeed obtained.

\section{Discussion}

From a biological point of view it is important to examine the roles which the various parameters play in determining the shape and size of the respective waves. The regular perturbation solution obtained in the previous section lends itself particularly well to such an exercise as it is possible to predict the qualitative role of some of the model parameters from the perturbation solution. It is also relatively easy to confirm these predictions by numerical studies.

We begin by investigating how the epidermal contraction wave is influenced by the adhesion tethers with which the epidermal sheet is connected through the basal lamina to the dermal layer. For large values of $\rho$, the sheet is firmly attached and the dermal travelling wave causes a very small disturbance in the epithelial layer. This is apparent from the scaling (4.16) we used, since by increasing $\rho$ the value of $\Sigma$ decreases. On the other hand, decreasing the value of $\rho$ has the opposite effect - the amplitude of the epithelial contraction wave increases.

It is interesting to note that when we do not have epithelial tethering, that is, $\rho$ is zero, two constants appear when we integrate the epidermal equation, (2.4a),

$$
\mu \frac{\partial \theta}{\partial t}+\theta+\frac{\tau}{1+v(1-\theta)}=k_{0}(t) x+k_{1}(t) .
$$


This equation does not possess travelling wavefront solutions with constant speed and shape. Due to the terms on the right hand side, the equation does not convert into an ordinary differential equation in terms of $z$ after the usual substitution $z=x+v t$.

Changing the traction parameter, $\tau$, has a similar effect as changing $\rho$. For example, by decreasing the epithelial traction parameter $\tau$, the value of the scaling parameter $\Sigma$ in (4.16) decreases and so does the size of the epithelial contraction wave.

The value of the viscosity parameter, $\mu$, similarly influences the amplitude of the epithelial contraction wave. When $\mu$ is very large, say $O\left(1 / \varepsilon^{2}\right)$, the viscous nature of the epithelial sheet dominates the elastic properties. The size of the contraction wave decreases accordingly, since now our $O(1)$ perturbation solution is $\Theta_{0}=0$. On the other hand, by decreasing the viscous parameter, $\mu$, to say $O(\varepsilon)$, the $\mu \Theta_{0}^{\prime \prime \prime}$ term, see Fig. $3 b$, in our $O(\varepsilon)$ solution (4.26) disappears. The disturbance in the leading edge of the dilation wave therefore increases while that in the trailing edge decreases. The change in the solution, however, would be very small, since decreasing $\mu$ only effects the first and higher order terms in our perturbation solution.

Hence the results obtained here lead to several biological predictions. To summarize: a change in epithelial viscosity, traction and/or adhesion influences the amplitude of the contraction wave.

These predictions could be tested experimentally. For example, by inducing a travelling wave in the dermal layer one could then examine the epidermal layer for a disturbance wave as seen in the solutions considered above. A dermal cell density wave could, for example, be initiated by injecting a mitotic promoter at one end of the developing skin. One could then investigate whether the amplitude of the epithelial wave increases if, say, the tethers, with which the epithelial layer is attached to the dermal layer, are severed. Such an experiment could also indicate whether the dermalepidermal interaction is chemical or mechanical.

Biologically it is believed that the whole pattern formation process in morphogenesis is the result of a few simple mechanisms. In Cruywagen et al. (1992) we showed that this tissue interaction mechanism could generate synchronous and sequential spatial patterns in one- and two-dimensional domains. (See also Cruywagen et al. 1993, where the pattern is shown to depend on initial conditions.) Here, we have illustrated that tissue interaction models can exhibit travelling wave solutions in one-dimension. Our simulations also show that the model can exhibit travelling wave solutions which leave behind a spatial pattern (Cruywagen et al. 1994). The versatility of this mechanism thus lends additional support to the key role of tissue interaction in morphogenesis. 
Applied Mathematics, University of Washington, Seattle for its hospitality, and for support from the Robert F. Philip Endowment.

\section{References}

Cheer, A., Nuccitelli, R., Oster, G. F., Vincent, J.-P.: Cortical activity in vertebrate eggs. I. The activation waves. J. Theor. Biol. 124, 377-404 (1987)

Cruywagen, G. C., Maini, P. K., Murray, J. D.: Sequential pattern formation in a model for skin morphogenesis. IMA J. Math. Appl Medic. Biol. 9, 227-248 (1992)

Cruywagen, G. C., Maini, P. K., Murray, J. D.: Sequential and synchronous skin pattern formation. In: Othmer, H. G., Maini, P. K., Mrray, J. D. (eds.) Proceedings of the NATO ARW on Biological Pattern Formation, NATO Conference Series. New York: Plenum Press (in press)

Cruywagen, G. C., Murray, J. D.: On a tissue interaction model for skin pattern formation. J. Nonlin. Sci. 2, 217-240 (1992)

Cruywagen, G. C., Maini, P. K., Murray, J. D.: Bifurcating spatial patterns arising from travelling waves in a tissue interaction model. Appl. Math. Lett. 7(3), 63-66 (1994)

Fife, P. C.: Mathematical aspects of reacting and diffusing systems. Lect Notes in Biomathematics 28. Berlin Heidelberg New York: Springer 1979

Kolmogoroff, A., Petrovsky, I., Piscounoff, N.: Étude de l'équation de la diffusion avec croissance de la quantité de matière et son application à un problème biologique. Moscow Univ. Bull. Math. 1, 1-25 (1937)

Lane, D. C., Murray, J. D., Manoranjan, V. S.: Analysis of wave phenomena in a morphogenetic mechanochemical model and an application to post-fertilisation waves on eggs. IMA J. Math. Appl. Medic. Biol. 4, 309-331 (1937)

Mollison, D.: Spatial contact models for ecological and epidemic spread. J. Roy. Stat. Soc. B39, 283-326 (1977)

Murray, J. D.: Nonlinear differential equation models in biology. Oxford: Clarendon Press 1977

Murray, J. D.: Mathematical biology. Berlin Heidelberg New York: Springer 1989

Murray, J. D., Cruywagen, G. C.: Threshold bifurcation in tissue interaction models for spatial pattern generation. Trans. R. Soc. Lond. A (in press)

Murray, J. D., Cruywagen, G. C., Maini, P. K.: Pattern formation in tissue interaction models. In: Lecture Notes in Biomathematics 100 Levin S. (ed.) Berlin Heidelberg New York: Springer (in press)

Murray, J. D., Oster, G. F.: Generation of biological pattern and form. IMA J. Maths. Appl. Med. Biol. 1, 51-75 (1984) 